\title{
La organización de los cuidados, más allá de la dicotomía entre esfera pública y esfera privada
}

The organization of care. Beyond the dichotomy between public sphere and private sphere

\author{
Chiara Cerri \\ Centro de Ciencias Humanas y Sociales. Consejo Superior de Investigaciones Científicas (CSIC). Madrid (España) \\ chiara.cerri@cchs.csic.es
}

\author{
Laura Alamillo-Martínez \\ Departamento de Antropología Social, UCM. Madrid (España) \\ laura.alamillo.ucm@gmail.com
}

\begin{abstract}
RESUMEN
A través de una revisión de conceptos desarrollados principalmente en relación al feminismo y al género desde distintas disciplinas (antropología, economía, sociología, filosofía, psicología), el artículo pretende mostrar la complejidad de la noción de cuidado y su utilidad como herramienta teórica para superar las fronteras entre las esferas privada y pública. A lo largo del texto se plantea que el cuidado no es exclusivo del ámbito familiar o doméstico, sino que se configura como una necesidad de todas las personas, por naturaleza interdependientes. De ahí la importancia de presentar un corpus teórico que permita orientar la mirada etnográfica hacia cómo se configuran las relaciones de cuidado en contextos específicos, para que aspectos aparentemente banales sean tomados en consideración.
\end{abstract}

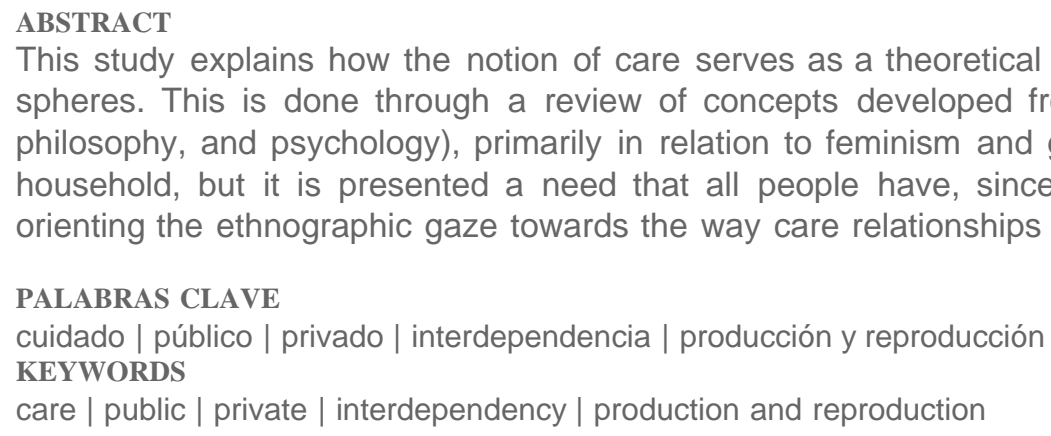

PALABRAS CLAVE

cuidado | público | privado | interdependencia | producción y reproducción KEYWORDS

care | public | private | interdependency | production and reproduction spheres. This is done through a review of concepts developed from different disciplines (anthropology, economics, sociology, philosophy, and psychology), primarily in relation to feminism and gender. It is shown that care is not unique to the family or household, but it is presented a need that all people have, since we are naturally interdependent. Hence the importance of orienting the ethnographic gaze towards the way care relationships are shaped in specific contexts.

\section{Introducción}

El objetivo de este artículo es mostrar de qué manera la noción de cuidado se configura como una herramienta teórica relevante para la superación de la dicotomía entre esfera pública y privada y, de esta manera, para una organización más equitativa de la sociedad. Al mismo tiempo, es nuestra intención ofrecer un corpus teórico lo más amplio posible sobre el cual se pueden apoyar futuras investigaciones antropológicas. Para ello, llevaremos a cabo una revisión de conceptos desarrollados principalmente en relación al feminismo o al género desde distintas disciplinas (antropología, economía, sociología, filosofía, psicología), planteando que el cuidado no es exclusivo de la esfera familiar o doméstica, sino que se configura como una responsabilidad social asumida por distintos agentes, que contribuye notablemente al bienestar de las personas.

Por razones de extensión no profundizaremos en la diversidad de posturas que existen respecto al género y el feminismo (1). Nuestra propia manera de situarnos es la siguiente: entendemos por feminismo un conjunto de movimientos sociales e intelectuales que aspiran y trabajan para alcanzar la igualdad de derechos y oportunidades entre hombres y mujeres. Consideramos que las teorías del 
género son fundamentales en la medida en la que han mostrado que las categorías de "mujer" y "hombre" son construcciones sociales específicas a cada contexto socio-cultural que cargan con significados y expectativas diversos rasgos físicos.

En el caso de España la familia sigue siendo considerada el principal agente proveedor de cuidado y servicios para el bienestar (Durán 2002), a pesar de los cambios impulsados por la incorporación generalizada de las mujeres al mercado de trabajo y de las dificultades de cuidar por las escasas medidas existentes para lograr que varones y mujeres participen satisfactoriamente en su vida familiar y laboral. También ha habido una generalización de agentes como el tercer sector y el mercado encargados de proveer cuidado, lo que ha conllevado cambios simbólicos y socio-económicos. Simbólicos porque permiten considerar el cuidado no solo como una tarea exclusivamente familiar y "naturalmente" femenina; socio-económicos porque el cuidado deja de ser un trabajo eminentemente no remunerado.

Sin embargo, dichos cambios siguen sin ser reconocidos por parte de la sociedad general y sobre todo por parte de los líderes políticos de España (de ahí que en época de recortes se haya atacado a la educación, la sanidad y los servicios de atención a la "dependencia"). Es cierto también que la aparición de los "nuevos agentes" (mercado y tercer sector) ha ocasionados a veces unos cambios perjudiciales en vista de una organización más equitativa de la sociedad. En el caso del mercado, por ejemplo, donde la tendencia es mercantilizar los cuidados para lucrarse en lugar de respetarlo en tanto que necesidad humana fundamental, existe el riesgo de aumentar la distancia entre aquellos que pueden "permitirse" contratar y comprar servicios y aquellos que no pueden y, en el caso de que los servicios públicos sean insuficientes, tienen que remitirse a la "voluntariedad" de los demás. Asimismo, a veces se ha provocado la expansión de un mercado en economía sumergida y de las llamadas cadenas internacionales de cuidado (Comas d'Argemir 2009).

Existen, pues, importantes razones sociales por las cuales creemos necesario profundizar en la cuestión del cuidado. En este sentido, la reflexión que planteamos, fundamentalmente teórica, puede ayudar a informar la mirada etnográfica, de tal manera que acontecimientos aparentemente banales adquieran interés y relevancia en tanto que cuestiones sociales.

Con el mismo propósito de explicitar la complejidad de las relaciones de cuidado que se refleja en la práctica, a lo largo del texto pondremos algunos ejemplos, algunos abstractos e hipotéticos, otros más empíricos sacados de las etnografías de las autoras. En efecto, la propuesta teórica que aquí exponemos surge de la necesidad de dar sentido a observaciones recurrentes en nuestros trabajos de campo, realizados en un caso en contextos de atención a la tercera y cuarta edad (2) y en otro caso en contextos educativos con adolescentes (Alamillo y Cerri 2012).

Primero, para mostrar en qué circunstancias se ha gestionado la (in)visibilidad del cuidado, haremos un recorrido por los cambios sociales que han dado lugar a la construcción cultural de dos esferas, una considerada pública y masculina, encargada de la producción, y otra privada y femenina, encargada de la reproducción. A continuación, expondremos algunos planteamientos que visibilizan como las labores de reproducción también tienen lugar en el ámbito público a través de su mercantilización y externalización: un proceso de cambio social, relacionado con el fenómeno del individualismo institucionalizado (Beck y Beck- Gernsheim 2002), por el que las personas acuden a servicios públicos o contratan servicios externos para la realización de labores que anteriormente se desarrollaban en el espacio del hogar, considerado privado (la educación, las curas, la producción de ropa y comida, etc.).

Después de este recorrido teórico, que muestra la construcción histórica y cultural de las esferas pública y privada, así como de los conceptos de "familia", "hogar" y "grupo doméstico", el artículo se centrará en la noción de cuidado y en las nociones relacionadas de dependencia y vulnerabilidad.

Para concluir, expondremos las propuestas, hechas principalmente desde la ética del cuidado, para promover cambios en la organización social: poner el cuidado y el bienestar de las personas en el centro de la vida social, moral y política, para superar así la dicotomía entre público y privado y, al mismo 
tiempo, la dicotomía entre sujetos independientes y sujetos dependientes.

El objetivo de esta propuesta es entonces ofrecer un corpus teórico para que futuras investigaciones tengan en consideración cómo se relacionan las teorías sobre la reproducción con las teorías del cuidado y, apoyándose en los planteamientos teóricos, den lugar a un material empírico con el cual también poder reclamar políticas públicas que asuman el cuidado como responsabilidad social y no como asunto que deba resolverse a título individual.

\section{Cambios en la organización y el reconocimiento social de los trabajos que se realizan en los hogares}

Si bien en este artículo vamos a hablar principalmente de cuidado, conviene resaltar que su organización es fruto de convenciones sociales, de ahí la importancia de llevar a cabo un pequeño repaso a cómo se ha gestionado en distintos tiempos y lugares e incluso a cómo ha sido estudiado teóricamente. Para situarnos, en términos generales podemos decir que el cuidado engloba todas aquellas actividades que se realizan para el bienestar físico, psíquico y emocional de las personas (Comas d'Argemir 1993). Sin embargo, como veremos a lo largo del artículo, el concepto de cuidado es mucho más amplio y complejo, dependiendo también de las diferentes perspectivas teóricas desde las cuales se mira.

No obstante, parece un hecho generalizado que cuando se habla de cuidado, se piensa en la familia. Por ejemplo, en cómo se reparten las tareas entre los miembros del hogar o en cómo las familias que pueden permitírselo contratan a mujeres para que se hagan cargo de cuidar a sus miembros. Por ello, en este apartado nos centraremos concretamente en algunas nociones básicas respecto a los cambios que se han dado en la organización familiar del trabajo.

La antropología estudia desde sus inicios distintas formas de parentesco, que "responde a la necesidad de los grupos humanos de garantizar su supervivencia ya que, en base al mismo, se han organizado y se organizan en numerosas culturas aspectos tales como la reproducción biológica y social, la división sexual de tareas, la organización de la residencia, la nutrición o el consumo" (Pichardo 2009: 154). No podemos ocuparnos de todas las formas de parentesco (3), de modo que aquí nos centramos en cómo se organiza el parentesco en los países industrializados o, dicho más informalmente, en lo que concierne a la familia.

Cuando se habla de familia se suele hacer referencia a "un grupo de personas emparentadas por lazos de afinidad (derivados de la díada conyugal o pareja) y de consanguinidad (tanto ascendientes como descendientes)" (Rivas et al. 2009: 29). Otra forma de definir la familia es a través de sus funciones. Entre ellas se cuentan "la crianza, los cuidados, la producción, el consumo, la socialización, la sociabilidad" (Rivas et al. 2009: 29), la procreación y la socialización entendida como la "transferencia de conocimientos y la introducción en las redes sociales" (Narotzky 2004: 227), y el aprendizaje de las relaciones de género (Willis 2007: 433). Esas funciones se organizan de distintas maneras en las diferentes sociedades, de modo que desde el feminismo se ha propuesto entender el concepto de familia como ideología. Una ideología que, sobre todo, "determina las relaciones de género y la subordinación de la mujer" (Berk 1985; Ferree 1990 en Rivas et al. 2009: 21).

Además, el concepto de familia aparece íntimamente unido al concepto de hogar, entendido este como "la unidad corresidencial donde las personas se encuentran empíricamente" (Ferree 1990, en Rivas et al. 2009: 22) y al de grupo doméstico, que hace referencia:

"al conjunto de personas que comparten un mismo espacio de residencia, siendo éste un espacio de trabajo y producción, así como también un espacio de descanso, de convivencia, y de consumo, por tanto, de reproducción. [...] el grupo doméstico puede incluir personas sin relación de parentesco, que comparten las actividades de producción (obreros, aprendices, etc.) o que no las comparten (inquilinos, huéspedes, etc.)" (Rivas et al. 2009: 29). 
Combinando las anteriores definiciones, nos encontramos con que la familia es, en palabras de Narotzky, un "constructo ideológico" (Narotzky 2004:168), que funciona reclutando personas en los hogares y que contribuye a regular las relaciones sociales tanto dentro de los mismos, como con el entorno social. Es más, la ideología sobre la familia "puede considerarse un medio de garantizar y ocultar relaciones de poder y explotación en el seno de los hogares" (Rapp 1978 y 1987, en Narotzky 2004: 171).

Tanto la noción de familia como la de hogar modificaron su significado a raíz de los cambios en la organización social que supuso la industrialización en los países europeos. Así, hasta el siglo XVIII se entiende el hogar como un "grupo de personas unidas por lazos de parentesco u otros, que comparten un espacio y organizan procesos de consumo, distribución y producción" (Narotzky 2004: 168). Es decir, se consideran parte de la familia aquellas personas que no están vinculadas por consanguinidad ya que lo fundamental de los hogares es que en ellos se desarrollan los trabajos necesarios para el bienestar de sus miembros (desde el cultivo a la confección de la ropa y la comida).

Durante el siglo XIX se extiende entre la burguesía "la idea de familia como grupo estrictamente limitado a las relaciones de parentesco más cercanas y funcionalmente orientadas a la procreación y a la socialización" (Narotzky 2004: 168-169). En otras palabras, se desvinculan los procesos productivos de la noción de familia y se limita esta expresión a las personas biológicamente emparentadas. ¿Cómo acontecen estos dos procesos?

Por un lado, dentro del hogar la "intimidad" se usa para explicar la distinción entre familiares y sirvientes, inquilinos, etc. Por otro lado, "los procesos de producción se distinguen cada vez más de las tareas relacionadas con el hogar" (Narotzky 2004: 169). Así, se produce una separación entre el espacio público, al que se atribuye la "producción", y el espacio privado-doméstico, donde se llevan a cabo los trabajos de "reproducción". Simultáneamente, el "trabajo" se define como actividad extra-doméstica, invisibilizando y desvalorizando así el trabajo llevado a cabo en el hogar.

Martín Palomo (2008b) argumenta que antes de la industrialización era difícil saber qué miembro de la familia realizaba cada una de las actividades que hacían posible satisfacer las necesidades básicas del conjunto. En cambio, el proceso de industrialización da lugar a una separación de espacios y usos de los mismos. Así, se saca del ámbito doméstico la producción de bienes y servicios para el mercado y aparecen los salarios para los trabajos realizados fuera del ámbito doméstico.

Por tanto, si con anterioridad a la industrialización todo el grupo doméstico contribuía al bienestar de las personas que cohabitaban, a partir del siglo XVIII el reconocimiento social se centra principalmente en el mercado monetario y el trabajo asalariado, mientras que el trabajo doméstico, realizado en su mayor parte por mujeres, queda sin reconocimiento social. Al mismo tiempo, el trabajo doméstico se ve despojado de su valor a causa la extensión de la ideología que naturaliza, restándole importancia, el amor familiar y la capacidad de las mujeres de cuidar de otros, a la vez que enaltece el esfuerzo para mantener en funcionamiento el ámbito productivo.

En efecto, el trabajo doméstico realizado por las mujeres dentro del hogar pasa a ser considerado propio de la esfera de lo emocional, en contraposición a la racionalidad del empleo, perteneciente a la esfera pública y masculina. Se asume que existe algo así como un "contrato laboral doméstico" que sigue una lógica diferente a la del mercado competitivo: el trabajo se hace gratuitamente y pensando en el bienestar de los demás, procurando satisfacción emocional en lugar de una recompensa económica ponderada racionalmente. Este es el origen de la dicotomía público/privado, que da lugar a que se atribuyan distintos valores a estos espacios y a que los cuidados queden inscritos en las familias y vinculados a la feminidad y a lo doméstico (Martín Palomo 2008b).

En resumen, nos encontramos con que se da un proceso de alejamiento entre la vida laboral y la vida de las personas en el hogar, que ideológicamente se plasma en la separación de la esfera doméstica y la productiva, haciendo que la doméstica se configure como esfera esencialmente privada, en contraposición con la pública (Tronto 2009). Se establece entonces el predominio de la producción de bienes de cambio (Gregorio Gil 2005) sobre la reproducción y la producción de bienes de uso (Badgett y 
Podemos ver que hay dos movimientos que moldean la ideología sobre la familia:

"uno que separa los ámbitos de la producción y la reproducción -procreación, socialización, mantenimiento-, y otro que naturaliza y privatiza la familia nuclear. Ambos movimientos contribuyen a la idea de la familia como un conjunto de relaciones no económicas, altamente emocionales y de responsabilidades de género naturales que deberían respetarse para que prevalezca el orden moral natural" (Narotzky 2004: 169).

Mientras se atribuye a la esfera privada, y a las mujeres y niños que pertenecen a ella, la emotividad y la irracionalidad, "se presupone que las emociones y los sentimientos no son requeridos por la acción racional de los individuos o para el funcionamiento óptimo de las instituciones" (Martín Palomo 2008b: 24).

Esto queda plasmado en el hecho de que las teorías económicas clásica y neoclásica opongan el interés del individuo "por excelencia" (el agente económico racional) al "altruismo", que solo cabría en la familia (Badgett y Folbre 1999). Por tanto, según estas teorías, un trabajador remunerado que persigue su interés personal (el dinero) no puede "preocuparse" de manera altruista por las personas que reciben su servicio, ya que esto iría contra la lógica económica racional.

Pero, igual que sabemos que las emociones juegan un papel importante en el ámbito "público", también es ingenuo pensar que el trabajo doméstico es siempre puramente altruista. De hecho, en muchas ocasiones se trata de una imposición (en el campo de las relaciones de poder, es frecuente que las mujeres queden al cargo de los trabajos domésticos, igual que los jóvenes tienen que obedecer a sus mayores).

Las mencionadas teorías sobre el "actor racional" ignoran hechos observables en la etnografía. Por ejemplo, frente a la idea de que los trabajadores remunerados, en tanto que agente racionales en la esfera pública, no se preocuparían de las personas que reciben sus servicios, nos encontramos con casos en los que ciertas auxiliares en Centros de Días o trabajadoras en Centros de Mayores, a pesar de tener condiciones laborales precarias, añaden un plus de afectividad en sus labores. Entre los motivos que les llevan a añadir dicho plus se encuentran que "empatizan" con los mayores de los que se ocupan; que consideran que así el trabajo se realiza mejor y tiene efectos positivos para los mayores; que consideran que el cariño y el afecto es lo que más necesitan los mayores. Ninguna de estas razones tiene que ver con la búsqueda de un interés personal o económico, sino con la preocupación por las personas de su entorno.

Vemos entonces como el altruismo no es exclusivo de las familias, por lo que la idea de la familia como lugar de afectos y sentimientos, contrapuesta al mercado como lugar racional es una construcción cultural. Pero, en lugar de admitir la naturaleza culturar de dicha dicotomía (racional/emocional), la implicación personal de los trabajadores tiende a no ser considerada como una conducta profesional "adecuada": a menudo los profesionales de la educación o la sanidad plantean la necesidad de un cierto distanciamiento de sus alumnos o pacientes para poder llevar a cabo su trabajo.

\section{Superación del concepto de unidad doméstica y aparición del individualismo institucionalizado}

En el apartado anterior hemos distinguido entre familia, hogar y grupo doméstico, y hemos hecho referencia a cómo existe un vínculo entre el proceso de industrialización y externalización de la producción para el mercado y el de invisibilización y desvalorización de los trabajos reproductivos o de cuidados. Además de analizar la familia o el hogar en torno a los procesos de producción y reproducción, se puede poner el foco en los procesos de consumo. Narotzky (2004) por ejemplo denuncia que los modelos económicos se centran en el proceso de producción y no prestan una atención adecuada al consumo, que está más directamente referido a la subsistencia de las personas. Al mismo tiempo, la 
autora argumenta que los análisis económicos que toman al hogar como unidad de consumo tienden a homogeneizar ese espacio sin prestar atención a lo que ocurre dentro de este espacio doméstico, que se configura como "un haz de relaciones entre personas reunidas en torno a un complejo de consumo" (Narotzky 2004: 172). Dicha invisibilización de las relaciones dentro de los hogares es acentuada a causa de la idea de que cada hogar es una unidad, con unos límites bien definidos, por lo que analíticamente se tiende a aislar cada unidad de otras unidades similares.

A su vez, esto está relacionado con la idea del hogar como espacio de los afectos y del mercado como espacio libre de ellos, que funciona únicamente en base a criterios racionales. Narotzky lo expresa con estas palabras:

"los intercambios se conciben entre una unidad «interior» de consumo doméstico y un mundo «exterior» de procesos económicos «reales», es decir, de producción y distribución. Ideológicamente, se produce una confrontación entre lo moral y lo inmoral, entre actos afectivos y desalmados, entre los mundos emocional y racional. El concepto de unidad doméstica de consumo marca una frontera que divide dos espacios completamente diferentes, tanto material como simbólicamente" (Narotzky 2004: 172).

Esta manera de entender la unidad doméstica, basada en el consumo, también invisibiliza el trabajo delegado en las mujeres de gestionar los recursos para satisfacer las necesidades de los miembros de la familia o unidad doméstica. Aún más, invisibiliza la diversidad existente entre distintos hogares. Para empezar, simbólicamente, lleva a considerar el hogar:

"como una «economía moral» organizada de acuerdo a principios de reciprocidad, consenso y altruismo entre sus miembros (Grasmuk y Pessar 1991). También deja de lado aquellos grupos domésticos cuyos ingresos no eran puestos en común, sino controlados por los individuos que los generaban. Asimismo, ignora las jerarquías de poder existentes [...], especialmente, las que se basaban en el género y la generación" (Rivas et al. 2009: 28).

En otras palabras, en base a dicha perspectiva, sólo se consideran unidades domésticas aquellas en las que rige la lógica hegemónica de organización familiar.

Así, nos encontramos con que es necesario cambiar de enfoque para visibilizar el trabajo doméstico, expresión con la que se hace referencia "a la producción de bienes y servicios en el hogar, y al cuidado en sentido general" (Narotzky 2004: 208).

Narotzky propone estudiar las redes personales de consumo, porque permiten observar que la capacidad individual para obtener recursos depende no sólo de los miembros del hogar, sino de la "estructura social en general, y en particular el parentesco, la amistad y las redes de vecinos" (Narotzky 2004: 197). Es decir, hay que romper con la idea de unidad doméstica como unidad de bienes de uso y de consumo porque no alcanza a explicar la familia, ni es una entidad legítima para explicar el mantenimiento de la vida humana, ya que el consumo se organiza a nivel de redes sociales que desbordan la unidad doméstica.

Por ejemplo, algunas personas mayores acuden a servicios sociales públicos o a su red de vecindad para fomentar su bienestar. Igualmente, hay jóvenes que deciden consumir una cosa u otra en función del acceso que les facilite el grupo de pares.

Esto legitima estudiar los cuidados que se desarrollan fuera del hogar. En primer lugar porque, como nos recuerda Carrasco, "las necesidades humanas son de bienes y servicios pero también de afectos y relaciones" (Carrasco 1998: 3). En segundo lugar, porque dichas necesidades no siempre son satisfechas en exclusiva dentro del hogar.

El hecho de que a nivel teórico se hayan empezado a denunciar las desigualdades dentro de los hogares y el papel de las redes personales, tiene que ver con otro proceso social que se incrementa con posterioridad a la generalización de la industrialización y en relación al desarrollo de los Estados de 
bienestar, que a su vez expresa ciertos ideales de la Modernidad (4). Nos referimos al proceso de individualización o individualismo institucionalizado (Beck y Beck-Gernsheim 2002), es decir, las dinámicas que llevan a considerar a los individuos como unidades básicas de reproducción social.

El Estado de bienestar (entendido como aquel que asume como propia la responsabilidad del bienestar de sus miembros y por lo tanto garantiza el acceso de sus miembros a servicios esenciales como, por ejemplo, la educación, la sanidad y la justicia) promueve el individualismo institucionalizado en la medida en la que convierte a los individuos, y no a los grupos, en beneficiarios de sus servicios. Y esto es así porque estatalmente es el individuo quien es considerado como el lugar en el que se toman las decisiones sobre el estilo de vida, no los colectivos a los que pertenece (familia, vecindario, clase, Estado, etc.).

Los Beck destacan que la individualización (5) contribuye a la libertad individual e insiste en que eso no convierte al individuo en un ser independiente, simplemente, pasa a depender de otras instituciones (el Estado, el mercado, la burocracia...). Al mismo tiempo, el individuo siente que elige la vida que quiere llevar, elige sus lazos sociales y se hace a sí mismo, configurando una biografía electiva. De ahí que si las elecciones conllevan algún tipo de fracaso, éste sea tomado como propio y las situaciones en donde se sufre ese fracaso se silencien e invisibilicen, pues las personas experimentan culpa o vergüenza en lugar de conciencia de clase.

Los Beck (Beck y Beck-Gernsheim 2002) muestran el funcionamiento del proceso de individualización a través del caso de las mujeres. La escuela y la educación mixta han sido una de las vías principales de transmisión del mensaje de individualización a las mujeres: en ella se encuentran sometidas a las mismas pruebas y competiciones que los varones, ya que se las evalúa en función de sus resultados individuales. Otro de los efectos del proceso de individualización de las mujeres pasa por su incorporación al mundo laboral.

Lo que importa subrayar aquí es el hecho de que con el proceso de individualización de las mujeres se da un cambio también en el significado de la familia: las mujeres dejan de plantearse qué pueden hacer por la familia y empiezan a preguntarse qué puede hacer la familia por ellas.

De modo que, por un lado, los cambios que han afectado a la sociedad y a los roles de género han hecho que la familia pase de ser una comunidad de necesidades y de trabajo, cuidado y apoyo mutuo, a ser una comunidad de sentimientos, basada en la afinidad electiva (6). Por otro lado, la falta de implicación de los varones en los trabajos de reproducción no remunerados y el deseo de igualdad de las mujeres han dado lugar a la reducción de la natalidad y a la delegación de los cuidados. ¿Qué significa delegar los cuidados?

Como hemos visto, la incorporación de las mujeres al mundo laboral remunerado ha fomentado, entre otras cosas, la erosión (todavía en curso) de la hegemonía del modelo de familia biparental heterosexual con el varón al cargo de los aspectos económicos y la mujer al cargo de la casa y la familia. Sin embargo, a causa del escaso interés del mercado laboral por facilitar la conciliación entre vida laboral remunerada y la vida personal (trabajo domestico no remunerado y tiempo de ocio), cada vez menos familias pueden asumir los trabajos de cuidado necesarios para el bienestar de los miembros del hogar. Esta situación ha llevado a mercantilizar y externalizar una parte importante de los considerados trabajos domésticos (7).

Empleamos el concepto de "cuidados mercantilizados" para referirnos a aquellos cuidados que se remuneran, sean o no miembros de la red de parentesco quien los realiza (la persona que limpia la casa sin pertenecer a la familia o una joven que de clases a su prima a cambio de una remuneración). Con "cuidados externalizados" entendemos tanto los cuidados que se realizan fuera de la red de parentesco o fuera del hogar con o sin remuneración (la asociación sin ánimo de lucro que trabaja para el entretenimiento de personas mayores o la labor de alimentación que facilitan restaurantes y empresas de catering y de comida precocinada).

El mercantilizar los cuidados no es algo nuevo. Las familias que se lo podían permitir han acudido a 
menudo a contratar personas que se hiciesen cargo de las tareas domésticas. Lo que nosotras proponemos como novedad es estudiar de qué modo operan esos lazos sociales que trascienden la dicotomía público/privado en tanto que emplean a personas (trabajo remunerado, en teoría de la esfera de lo público) para trabajar en el hogar (esfera privada) e incluso fuera de él (por ejemplo, quienes acuden a cenar a restaurantes o piden comida para llevar a casa).

Según Carrasco (1998) no todos los trabajos domésticos son sustituibles en el mercado: "las necesidades humanas tienen lo que podríamos llamar una dimensión más objetiva -que respondería más a necesidades biológicas- y otra más subjetiva que incluiría los afectos, el cuidado, la seguridad psicológica, la creación de relaciones y lazos humanos, etc. aspectos tan esenciales para la vida como el alimento más básico" (Carrasco 1998: 3). La autora parece plantear que existe una diferencia insalvable entre el trabajo remunerado y el doméstico que hace que ciertos trabajos domésticos no sean mercantilizables.

"El trabajo destinado al cuidado de las personas del hogar tiene otro contexto social y emocional que el trabajo remunerado y satisface necesidades personales y sociales que no permiten una simple sustitución con producción de mercado. Implica relaciones afectivo/sociales difícilmente separables de la actividad misma y crea un tejido complejo de relaciones humanas sobre el cual de alguna manera se sustenta el resto de la sociedad" (Schafër 1995, Himmelweit 1995, Carrasco 1998: 5).

Las afirmaciones de Carrasco parecen ofrecer pues una imagen romántica de las relaciones que se producen dentro de la familia (basadas en el amor y la afectividad), pero queremos citar a Narotzky para recordar las implicaciones que tiene para las mujeres esa lógica de los afectos a la hora de repartir los trabajos domésticos:

"Por lo general, las mujeres están vinculadas al trabajo doméstico a través de una relación familiar muy personalizada, cargada de emociones. Las cualidades concretas específicas de la relación posibilitan que los bienes y servicios producidos para los miembros del hogar no deban pagarse, y que la fuerza de trabajo doméstico del ama de casa no compita en el mercado. Las transferencias unilaterales, «altruistas», de bienes y servicios se realizan bajo las etiquetas del «amor» y «sacrificio» y difícilmente resultan comparables. La segunda condición del «contrato» doméstico del ama de casa implica, primero, que quienes sean ineficientes no serán expulsadas de sus trabajos ni eventualmente de un «mercado laboral doméstico» espurio, y segundo, la fuerza de trabajo de los miembros del hogar que sí participan en el mercado laboral, no es más costosa. Es decir, no es más costosa si el ama de casa que la produce es menos eficiente. Más bien ocurre lo contrario" (Narotzky 2004: 213-214).

Al argumentar que los trabajos que se realizan en el hogar tienen solo un carácter emocional, se está reproduciendo el discurso que liga lo emocional a lo familiar. De allí que las reivindicaciones que se han hecho al Estado de bienestar, en particular por parte de grupos feministas, consisten en que este asuma la responsabilidad del cuidado necesario de todos y todas las ciudadanas.

\section{Dimensiones, formas, niveles y límites del cuidado}

En los anteriores apartados hemos visto como, a partir del siglo XVIII, con el proceso de industrialización y, más recientemente, con el proceso de individualización e individualismo institucionalizado (Beck y Beck-Gernsheim 2002), ha tenido lugar una serie de transformaciones en el seno de los hogares, las familias, el parentesco y la relación entre trabajo remunerado y trabajo no remunerado.

En esta segunda parte del artículo nos centraremos en el cuidado, mostrando su relevancia en tanto que herramienta analítica para comprender la sociedad. En primer lugar mostraremos el alcance teórico del concepto de cuidado y en el segundo reflexionaremos sobre la necesidad de poner el cuidado en el centro del sistema social para el mantenimiento del bienestar colectivo e individual. 
A partir de los años ochenta, tras haber mostrado el carácter cultural, la importancia social y el valor económico del trabajo realizado por las mujeres en el ámbito doméstico y de forma no remunerada, la reflexión feminista se centra más en el cuidado. Su intención es dar cabida en los análisis a los aspectos más morales, afectivos y emotivos de los trabajos domésticos.

Inicialmente el debate académico se desarrolla en ámbito anglosajón con la introducción de la noción de care, que se caracteriza por tener una amplia variedad de significados: se refiere a los aspectos más materiales del cuidado, a los más sentimentales y emotivos, a la responsabilidad que entraña, la necesidad, etc.

En España algunos estudios prefieren distinguir entre "atención", "tarea" y "cuidado" (Precarias a la Deriva 2005, en Vega Solís 2009) para respetar los diferentes sentidos del care. Sin embargo, en este artículo utilizamos el término cuidado apoyándonos en la extensa producción científica y en el debate académico en lengua castellana que permiten concebir el "cuidado" como noción polisémica en consonancia con el término inglés de care.

Pero ¿qué es el cuidado? Fisher y Tronto lo definen como:

"una actividad de especie que incluye todo aquello que hacemos para mantener, continuar y reparar nuestro «mundo» de tal forma que podamos vivir en él lo mejor posible. Ese mundo incluye nuestros cuerpos, nuestros seres y nuestro entorno, todo lo cual buscamos para entretejerlo en una red compleja que sustenta la vida" (Fischer y Tronto 1990, en Tronto 2005: 3).

Se denota entonces que el cuidado es un concepto de difícil definición, o de definición muy amplia. Esto se debe a su complejidad y a la complejidad de las relaciones humanas en las que se desarrolla. Como hemos afirmado anteriormente, podemos apoyarnos en la definición de cuidado que da Comas d'Argemir (1993): el conjunto de todas aquellas actividades que se realizan para el bienestar físico, psíquico y emocional de las personas.

Sin embargo, para un análisis más detallado, podemos distinguir diferentes dimensiones del cuidado, tal como hace Tronto (8) $(2005,2009,2010)$, como el "cuidado práctico" (Taking care of (o Caring for y Care giving) y el "cuidado emotivo" (Caring about).

El práctico se refiere a las actividades de cuidados más materiales y corporales, como cocinar, limpiar, dar de comer, dar una clase, contar un cuento, etc., e implica asumir la responsabilidad del trabajo que hay que hacer para cuidar.

El segundo se refiere a los aspectos más emocionales del cuidado: dar y recibir cariño, afecto, apoyo moral y psíquico, etc. Un "preocuparse" de las personas que implica el reconocimiento de la necesidad de los demás.

Tronto (2009) argumenta que no toda actividad humana está relacionada con el cuidado, ya que solamente existe cuidado cuando la práctica (realizar cuidados) se une a la disposición (considerar las necesidades de los demás). Al mismo tiempo, por un lado alerta contra el considerar la disposición y los sentimientos como los únicos aspectos del cuidado, ya que concebirlo solo como un "trabajo de amor" Ileva a restablecer los estereotipos que asocian el cuidado con el trabajo femenino, "sentimentalizándolo" y limitando su valor. Por otro lado, sugiere que si no se incorpora al cuidado "práctico" una actitud afectiva puede darse un "mal cuidado". Aun así, dice Tronto, el "mal cuidado" es propio del cuidado. ¿Qué significa que un mal cuidado sea propio del cuidado? Lo podemos entender mejor acudiendo a Kittay (2002) que señala que:

"como actitud, el cuidado (caring) denota un lazo positivo, afectivo y una inversión en el bienestar de los demás. El trabajo de cuidado (care) puede ser realizado sin la actitud apropiada. Sin embargo, sin la actitud propia del cuidado (care), la disponibilidad al otro, 
esencial para comprender qué necesita, no es posible. Esto quiere decir que el trabajo que no está acompañado por la actitud del cuidado (care) no puede ser un buen cuidado (care)" (Kittay 2002, en Garrau y Le Goff 2010: 259-260).

Pascale Molinier, en cambio, afirma que si la actividad material de cuidado es realizada sin la actitud necesaria, no hay cuidado: "O hay cuidado (trabajo/actitud) o no lo hay. [...] El ser y el hacer son aquí indisociables" (Molinier 2009: 436).

Por su parte, siguiendo con las dimensiones y formas de cuidado, Martin argumenta que:

"la noción de care esconde diferentes niveles: individual, relacional, colectivo e institucional. Se puede leer en términos de relación, de actores, de prácticas y de dispositivos. El care representa a la vez una dimensión privada (en el sentido de la vida privada) y una dimensión pública, se parece a la vez a un deseo y a una responsabilidad, toma las formas de prácticas remuneradas o no, puede ser dispensado de manera informal o formal" (Martin 2008).

Es importante matizar que, aunque para el análisis es conveniente realizar distinciones entre las diferentes fases, dimensiones y niveles del cuidado, en la vida real las fronteras no son tan nítidas. La relevancia del cuidado como herramienta analítica radica exactamente en su capacidad para mostrar la continuidad entre dos esferas que se han postulado como diferenciadas, la pública y la privada, ya que el cuidado puede darse en ambas esferas al mismo tiempo. Por tanto las dimensiones, formas y niveles del cuidado no son "divisiones" que se oponen entre sí.

Vemos entonces que el concepto de cuidado conserva unas dificultades semánticas que impiden encontrar un acuerdo para una definición común. Sí hay acuerdo en considerar como punto de partida de los estudios sobre cuidado la publicación de In a different voice de la psicóloga Carol Gilligan (1982), en la que la autora presenta su teoría de la "ética del cuidado". Se trata de una ética propia de una "voz diferente" (9) que se contrapone a la "ética de la justicia" desarrollada por Kohlberg (1963, 1981). Este, psicólogo del desarrollo moral, realizó estudios con informantes varones y llegó a la conclusión de que los principios morales son principios universales y necesarios para el desarrollo cognitivo y moral de todos los individuos. Además, según esta teoría, la responsabilidad hacía los otros se configura como una "limitación de la acción" para evitar la posible agresión de los derechos de los demás: "no hacer algo" que pueda dañar los otros.

La ética del cuidado, al contrario de este tipo de teorías cercanas a las filosofías morales universalistas, plantea la importancia de las relaciones entre el Yo y el Otro para la sostenibilidad de la vida. No solo toma en consideración las particularidades de los individuos, considerados interdependientes, sino también los detalles de la situación concreta en la que se encuentran y que llevan a actuar de una forma $\mathrm{u}$ otra, considerando algo como justo o injusto. Al mismo tiempo, interpretando el mundo como una red de relaciones donde el uno depende del otro, la ética del cuidado reconoce la responsabilidad que cada uno tiene hacia los demás y la define como un deber moral de actuar (10). No hacerlo, podría ser inmoral (11).

Aunque en muchos casos el cuidado es más bien una obligación que una libertad, sobre todo en el caso de las mujeres, desde la óptica de la ética del cuidado este no es una carga, sino una responsabilidad que cada uno tiene con los demás, sean estos individuos cercanos o no (Saillant y Gagnon 2001) y sean estos concebidos como frágiles o no.

La ética del cuidado de Gilligan resulta entonces fascinante porque pone en evidencia la importancia de la interdependencia (Fine y Glendinning 2005) en las relaciones humanas y entiende el cuidado como una actividad y una responsabilidad social y moral, implícita en las vivencias y necesaria para la continua instauración y el mantenimiento de las relaciones sociales.

En este contexto, Tronto (2011) avisa del peligro escondido en la idea del cuidado como acción necesaria para preservar las relaciones sociales, ya que se pregunta si la instauración y la preservación de un conjunto de relaciones sociales son siempre tan "convenientes". Según ella habría que preguntarse 
qué relaciones son realmente sanas y merecen ser conservadas y, al mismo tiempo, considerar la imposibilidad de cuidar de todas las personas: "habrá un cierto número de personas o de preocupaciones de los que no nos podremos ocupar. Sin embargo, podríamos preguntarnos si nuestra ausencia de cuidado nos libera de toda responsabilidad moral" (Tronto 2011: 65). Es decir, según Tronto habría que preguntarse siempre cuáles son los límites satisfactorios de nuestro cuidado, para que este no se convierta en una justificación conservadora que nos lleve a cuidar solo de las personas cercanas y de nuestro entorno (nuestra familia, nuestros afiliados, nuestro país, etc.). Por esto, hay que prestar atención a que la elección de algunas relaciones, de las que cuidamos, no nos lleve a excluir otras. La solución, dice, consiste en precisar cómo transformar las instituciones políticas y sociales para que el cuidado sea puesto en el centro de la sociedad.

Por su parte, Paperman (2011) sostiene que si bien la dificultad de ampliar el cuidado a los demás en general, a aquellos con los que casi no tenemos relación, es una crítica constante que se hace a la ética del cuidado, esta no es la cuestión principal en la que hay que centrarse.

\begin{abstract}
"Aunque las relaciones de cuidado son características de las relaciones que mantenemos con las personas más cercanas, eso no significa que la proximidad y la densidad de estas relaciones configuren los rasgos más pertinentes para comprender lo más importante. El cuidado no es tanto la particularidad distintiva de las relaciones densas o de las relaciones con las personas más cercanas [...] como la respuesta a las exigencias que caracterizan las relaciones de dependencia. En este sentido, el problema central que plantea la ética del cuidado no es la posibilidad de ampliar el cuidado a los demás en general, sino y en primer lugar el reconocimiento de la realidad de nuestras dependencias" (Paperman 2011:328).
\end{abstract}

Se trata pues de concebir el cuidado como parte de la vida humana y asumir la vulnerabilidad y la dependencia de todas y todos como elementos constitutivos de la existencia y experiencia humana (Pérez Orozco y López Gil 2011).

El cuidado deja entonces de ser percibido como una actividad unidireccional, donde un cuidador "activo" independiente hace algo por un receptor "pasivo" dependiente y se presenta como una actividad que necesita de relaciones entre más de dos individuos.

\begin{abstract}
"comprender la importancia del cuidado para la vida humana [...] supone reconocer de manera más «realista» (en el sentido del realismo ordinario, no del realismo moral en general) de como lo hacen las teorías sociales y morales mayoritarias, que la dependencia y la vulnerabilidad no son unos accidentes que se presentan en el camino y que les llegan solo a los «otros», cualquiera esos sean: son rasgos característicos de la condición de todo el mundo, aunque los más favorecidos tienen la capacidad de atenuar o negar su intensidad" (Paperman y Laugier 2011: 24).
\end{abstract}

En el caso de los mayores, por ejemplo, es casi imposible definir con exactitud quien es el cuidador y quien es el cuidado. Cuando nos encontramos con madres de 80 o 90 años que, aunque tengan problemas de salud y acudan a los servicios públicos para recibir cuidados, siguen cuidando de los hijos o hijas que tienen en casa ¿cómo definimos quién es cada cual? ¿Quién es la persona dependiente y quien la independiente? No podemos hacerlo basándonos en la edad. Ni siquiera basándonos en los problemas de salud. Por tanto, aunque considerando el rol de "cuidadora infinita" que se asigna a las madres, este pequeño ejemplo demuestra como todos somos por naturaleza interdependientes.

\title{
5. De la ética del cuidado a las propuestas de cambio político
}

La ética del cuidado argumenta pues que todas y todos somos interdependientes, por lo que el cuidado es una tarea necesaria en todos los ámbitos y en todos los niveles de la vida humana. Sin embargo, hay muchos cuidados y muchas dependencias que no vienen "nombradas", por lo que se invisibilizan. Esto es consecuencia de la estructura jerárquica de la sociedad que, en función de la edad, el género, las 
condiciones económicas y otras variables en cada contexto, ha llevado a considerar algunos individuos como dependientes y otros como independientes. Por ejemplo, los niños y los mayores son considerados dependientes porque requieren cuidados a causa de su edad y de sus necesidades "físicas". También una esposa-ama de casa, aunque sea la que cuida del marido y del hogar, es considerada "dependiente" porque "depende" del dinero del marido. Por el mismo razonamiento, un varón adulto y con empleo remunerado es considerado independiente a nivel económico y físico.

Pero si la interdependencia caracteriza nuestro estar en el mundo, todos y todas necesitamos cuidados. Por lo que podríamos plantear que el cuidado no es solamente lo que hacen los más "fuertes" (independientes, autónomos, capacitados, adultos, sanos, etc.) por los más "débiles" (frágiles, dependientes, no-autónomos, discapacitados, no-adultos, enfermos, etc.). Más bien es posible concebirlo como el conjunto de las distintas actividades que contribuyen a preservar las sociedades y los individuos.

En base a estos presupuestos, creemos necesario ampliar el alcance del concepto de cuidado y empezar a considerar como cuidados todos aquellos trabajos que promueven el bienestar de las personas y la reproducción de los grupos. Por ejemplo, también podrían considerarse trabajos de cuidado aquellas atenciones que los varones se dirigen entre sí, a pesar de ser considerados independientes, o a otros varones más «poderosos» (jefes, clientes acaudalados, personas que ocupan puestos de poder, etc.). Otro ejemplo podrían ser las relaciones que se establecen en un aula, donde los estudiantes "populares" son los que más atención y afectos reciben de sus compañeros, e incluso de los profesores. ¿Pueden este tipo de relaciones ser concebidas como un "autocuidado individualista" que se realiza como medio para conseguir unos intereses personales? En el caso del alumno popular, podemos interpretar que quien le cuida no está respondiendo a una necesidad del alumno popular sino a una necesidad propia de obtener una "recompensa": devenir popular también. Igual podríamos interpretar el caso de los hombres que cuidan de otros hombres "poderosos".

Sin embargo, también podemos interpretar estas relaciones como un cuidado «hacía arriba» y plantearnos que estas técnicas pueden redundar en beneficio personal precisamente porque quien está "arriba" en la escala social también necesita cuidado, y de ahí que recompense las atenciones a su persona. Volvemos así a la cuestión de la necesidad de cuidado de todos los seres humanos.

En este sentido Hochschild (2003) plantea que en la escala social los sentimientos positivos se dirigen hacia arriba, mientras que los negativos hacia abajo. Por este motivo, para las personas que están en la cima de la jerarquía social el mundo resulta un lugar benigno, mientras que para las personas que están en la base el mundo resulta un lugar hostil. De hecho, uno de los motivos para querer estar en la cima, es la necesidad de cuidado y, asimismo, una de las ventajas de estar más "arriba" en la escala social es la cantidad de atención que se recibe. Esto debería animarnos a visibilizar cómo se articulan las relaciones de cuidado con las relaciones de poder.

Por ejemplo, sería interesante que aquellas personas que trabajan desde la perspectiva del capital social además de investigar la densidad de las redes sociales de las personas o los efectos que las redes sociales tienen sobre la vida laboral o sobre la salud, investigasen también el modo como esas relaciones se establecen y cómo circulan los cuidados a través de las redes personales (por ejemplo, para demostrar la validez o la invalidad de las afirmaciones de Hochschild).

Asimismo, podemos por ejemplo prestar atención a la influencia de las estructuras de poder en las interacciones entre alumnos y profesores o en la relación entre mayores y trabajadores en un Centro de día, donde unos son los "usuarios" (alumnos y mayores) y los otros son los trabajadores que cuidan. Pero los usuarios también pueden cuidar de los trabajadores, aunque sea por diferentes motivos. En el caso de los alumnos puede ser porque cuidar del profesor significa recibir buenas notas, pero también puede ser porque el alumno entiende que el profesor necesita su atención para hacer bien su trabajo y sentirse satisfecho. En el caso de los mayores, estos no necesitan cuidar de los trabajadores para recibir buenas notas, pero pueden hacerlo por reciprocidad: si reciben la atención, práctica y afectiva, que consideran necesitar, responden a estos cuidado con otro cuidados. Pero también, en tanto que «clientes» de un 
servicio que están pagando, puede que lo reclamen sin cuidar del trabajador. Los ejemplos que muestran la complejidad del cuidado podrían ser infinitos: podemos tratar de descubrir si las mujeres tienden a cuidar más hacia "abajo" y los hombres más hacía "arriba" a causa de la educación de género recibida; también podemos analizar aquellos casos en los que hay personas que dejan de cuidar de sí mismas para cuidar de los demás: algunos ejemplos de esto serían las madres que se sacrifican por los hijos o los padres; los hijos e hijas con los padres o los hermanos y hermanas; el caso de los trabajadores remunerados que se involucran tanto en su profesión, para conseguir beneficios para la colectividad, que se olvidan de ellos mismos, algunos activistas, etc.

Todos estos ejemplos, más hipotéticos que etnográficos, nos llevan a distintas interpretaciones sobre las relaciones de cuidado. Para que dichas interpretaciones no se queden en simples especulaciones, como científicas sociales creemos necesario que se fomenten las investigaciones etnográficas acerca del cuidado para poner al descubierto los entresijos de las relaciones de cuidado en las sociedades.

Esto permitiría arrojar luz sobre qué es un buen o un mal cuidado; cuando se da o no se da cuidado; si es cierto, y si lo es por qué, que las relaciones de poder llevan a que se cuide con más gusto a los que están en el escalafón superior de la escala social; cómo y cuando las estructuras de poder, la reciprocidad y la educación de género influyen en las relaciones de cuidado. Además, permitiría, por un lado, que el estudio del cuidado no quede relegado a una dimensión puramente teórica y, por otro, revindicar nuevos mecanismos que garanticen la igualdad y la equidad en las sociedades.

Benhabib (1992) señala que "en las sociedades modernas [...] las cuestiones éticas de la justicia se han diferenciado de las cuestiones evaluativas de la vida buena, las relaciones y las obligaciones del cuidado y de la responsabilidad son cuestiones «personales» referidas a la autorrealización" (Benhabib 1992: 43). Así, el cuidado, en su amplio sentido, se distancia de las decisiones políticas que se centran únicamente en las personas que consideran "dependientes". Al contrario, creemos que el cuidado no puede ser considerado como una cuestión marginal referida a la «vida buena» y una cuestión personal diferente de las cuestiones "universales" de la justicia. Porque, como venimos argumentando desde el principio, el cuidado no es un comportamiento natural, responsabilidad sobre todo de mujeres (12), ni una producción "doméstica" o privada.

Siguiendo a Del Valle:

"el marco de valores que sustentaba tradicionalmente el cuidado ha experimentado cambios; la sociedad actual plantea contradicciones al potenciar simultáneamente el individualismo y las bases estructurales de dependencia de otras personas. La tensión que experimenta la familia entre autonomía y dependencia de sus miembros la sitúa en círculos más amplios: entre el individuo y el grupo familiar, entre lo biológico y lo social, entre lo privado y lo público" (Del Valle 2000: 21).

Por tanto, a nivel macrosocial hay que preguntarse de qué manera es posible repartir las labores necesarias para el bienestar de las personas entre el Estado, las familias, el mercado y la comunidad. A nivel microsocial, se trata de plantear la necesaria división de tareas de cuidado entre varones y mujeres y entre generaciones (Aguirre 2005).

El llamado "diamante del bienestar" (Evers, Pilj y Ungerson 1994, en Aguirre 2005) muestra cómo se reparte el cuidado de niños y mayores considerados dependientes entre cuatros agentes sociales principales (el Estado, el mercado, la familia y el voluntariado) y unos agentes "híbridos" como son los amigos, los vecinos, los servicios públicos a través de empresas privadas, ONGs subvencionadas por el Estado, etc.

Sin embargo, esta repartición entre los diferentes agentes del bienestar a veces conlleva desigualdades. Por ejemplo, si los servicios públicos son insuficientes para cubrir las necesidades de cuidado, aumenta la distancia entre aquellos que pueden "permitirse" contratar servicios de cuidado en el mercado y aquellos que no pueden. Además, en el caso del cuidado más práctico a menores y mayores, es notorio (Comas d'Argemir 2009; Pérez Orozco y López Gil 2011) que la introducción del mercado ha provocado 
en muchas ocasiones la expansión de un mercado en economía sumergida y de las llamadas cadenas internacionales de cuidado.

Asimismo, las organizaciones de voluntariado, a las que se remiten aquellos y aquellas que no tienen los medios suficientes para "comprar" cuidados en el mercado, a veces fomentan la precariedad del empleo profesional o, cuando acuden a voluntarios/as sin ninguna formación, una infra-profesionalización del trabajo de cuidar. También se dan casos en que dichas organizaciones de voluntariado preservan una visión vertical de las relaciones de cuidado sin plantear ninguna crítica constructiva de la sociedad y de las relaciones de poder: los "afortunados" son los únicos que pueden dar, mientras que los "desamparados" solo pueden recibir.

No es nuestra intención plantear que el voluntariado sea algo negativo, al revés, lo entendemos como una expresión del deseo humano de cuidar otras personas. Pero también creemos que para que no se fomenten todavía más desigualdades, es primordial que todos y todas vean respetado su derecho al cuidado.

Pérez Orozco y López Gil (2011) afirman que el derecho al cuidado incluye tres dimensiones: el derecho a recibir cuidado, el derecho a elegir entre cuidar y no cuidar, y el derecho a condiciones laborales dignas y justas en los trabajos de cuidado. A estas tres dimensiones se añade el derecho a negarse a recibir cuidado si estos son inapropiados (Anderson 2010).

Para que este derecho al cuidado venga respectado parece necesario, en primer lugar, un cambio de perspectiva que entienda el cuidado como una responsabilidad social, política y moral necesaria para la sostenibilidad misma de la vida humana y su entorno. De esta manera, poniendo el cuidado y el derecho al cuidado en el centro de las reflexiones y de las políticas, se podría poner en marcha una mejor repartición de las tareas entre todos los agentes de bienestar e ir hacía políticas que superen las dicotomías establecidas, que no son universales ni tienen los mismos contenidos allí donde existen (Del Valle 2004).

Según varias estudiosas (Garrau y Le Goff 2010, Daly y Lewis 2000, Martin Palomo 2009) no se trata de oponer lo público a lo privado, sino de desarrollar un sistema-red de agentes del cuidado que hagan disponibles los recursos y las oportunidades necesarias. Ir hacia un modelo de Social care a través de una reordenación de las obligaciones y de los derechos de todos los actores en juego y una integración de todas las actividades y relaciones implicadas en el sostenimiento de las necesidades físicas y emocionales, así como en los marcos normativos, económicos y sociales en los que se insertan.

Otras autoras (Carrasco 2003, Precarias a la deriva 2004) cuestionan este modelo porque parece esconder una estrategia dirigida a una refamiliarización y una privatización del cuidado, desplazando su coste otra vez hacia las familias, y las mujeres, o hacía el mercado.

Sin embargo, el modelo del Social care se presenta como una de las propuestas más sugerentes, porque concibe el cuidado como una responsabilidad y una necesidad compartida que va más allá del espacio privado y se expande hacia su entorno social más amplio (Tronto 2005, Saraceno 2005).

\footnotetext{
"Es necesario reconsiderar el problema de la separación entre público y privado. Esta separación contamina todo el pensamiento de la justicia llevando a una ceguera en la observación de las actividades y de los gestos necesarios para el mantenimiento de las personas y contribuye a que la justicia sea una conversación entre adultos «competentes» e iguales relacionados con los demás solamente de manera voluntaria, contractual y por asuntos específicos [...] Desde el punto de vista del cuidado, dicha concepción circunscribe artificialmente toda clase de relaciones sociales e políticas cubiertas por la sombra de la justicia [...] integrar el cuidado en un enfoque ético, social y político general, que no sea reservado a las mujeres sino que sea una aspiración para todos y por el mismo concepto de justicia" (Paperman y Laugier 2011:26).
}

La perspectiva de la ética del cuidado fomenta pues la reivindicación política de valorizar el cuidado y de 
reconfigurar las políticas públicas y las instituciones en función de esta valorización, para que se creen más condiciones de posibilidad (de cuidar o no cuidar, de recibir o no cuidado) que obligaciones impuestas (Pattaroni 2011).

Además, como sostiene Haber (2011), concebir el cuidado de esta manera significa concebirlo como "un tipo de trabajo fundamental que, como todos los demás trabajos, merece ser socialmente problematizado, justamente repartido, culturalmente respetado, y correctamente remunerado en algunos casos" (Haber 2011: 205).

Así, a nivel etnográfico, se hace necesario plasmar el modo en el que está articulado el cuidado en los contextos que estudiamos, para explicitar qué formas de cuidado están impuestas sobre qué agentes o por qué motivos se llevan a cabo qué formas de cuidado, o qué condiciones fomentan el altruismo a la hora de cuidar de los demás y qué condiciones inducen formas de no cuidado o cuáles fomentan la reciprocidad y cuáles la no reciprocidad.

\section{Conclusiones y nuevas preguntas de investigación}

En este artículo hemos querido mostrar los orígenes históricos y la naturaleza cultural de ciertas dicotomías establecidas y cómo a lo largo de ese proceso los cuidados han quedado relegados al ámbito de lo privado y asociados a las mujeres, invisibilizando la necesidad de asumirlos como responsabilidad social.

La reflexión llevada a cabo a partir de una revisión teórica nos ha permitido indagar acerca de los distintos niveles que influyen sobre la organización de los cuidados (moral, social, cultural, político, económico, etc.), insistiendo en que el cuidado no es exclusivo de la esfera familiar, o doméstica, y femenina. Para esto hemos mostrado las continuidades existentes entre privado y público, planteando que términos como dependiente e independiente, emocional y racional, reproducción y producción, trabajo no remunerado y empleo, etc. son artificios culturales que no reflejan la realidad en la que vivimos y en la que las relaciones de cuidado impregnan cualquier situación humana.

En este sentido, hemos indicado como las teóricas cercanas a la ética del cuidado insisten en la urgencia de fomentar una organización más horizontal de la sociedad, a través de unas nuevas perspectivas y propuestas que reivindican poner el cuidado y la sostenibilidad de la vida en el centro del sistema para visibilizar su alcance social, moral y político.

Al mismo tiempo, al tratar de ver la aplicación de las cuestiones teóricas en la práctica etnográfica, o a la hora de plantear propuestas políticas, hemos detectado situaciones respecto a las cuales nos surgen preguntas difíciles de responder. Aún quedan muchas cuestiones con las que lidiar. Por ejemplo el tipo de relaciones que se establecen entre las personas que pertenecen a redes sociales profesionalizadas, donde "mi profesora es cercana pero no puede ser mi amiga" y "la señora que limpia es casi como de la familia, pero no es de la familia", "el voluntario que viene a verme cada miércoles, ¿es mi amigo o no?"; la eterna disputa sobre si las escuelas son entidades de cuidado o educativas, si hay diferencias entre una y otra dimensión o si se pueden separar en absoluto; las consecuencias de la mercantilización de las relaciones de cuidado, en sus facetas positivas y negativas; las desigualdades existentes en el cuidado por motivos económicos.

A raíz de la crisis actual, tenemos múltiples ejemplos de cómo el Estado decide recortar el derecho de las personas a servicios públicos de calidad: recorte a los servicios de atención a la dependencia (13), recortes a los servicios de educación (14), cambios en la gestión de la sanidad (15), etc. Creemos por tanto que en estos momentos es nuestra responsabilidad profesional estudiar los efectos de este tipo de políticas. Por ejemplo, en España se considera la escolarización de las hijas como una medida que fomenta la conciliación personal y laboral de las madres y las niñas pasan una parte considerable de su tiempo en instituciones escolares, por lo que cabe preguntarse por las implicaciones que tienen los recortes respecto a este tipo de servicio, o medidas que permiten "abaratar los costes" al aumentar la 
ratio de personas que pueden estar al cargo de un profesional ¿cuál es el coste social de este ahorro económico?

Nos encontramos con ejemplos parecidos en el caso de los servicios de cuidado para mayores que se están viendo reducidos, cosa que en muchos casos lleva a que algunas personas se vean obligadas a abandonar sus puestos de trabajo para cuidar en casa de sus mayores, sin recibir ningún tipo de ayuda y asumiendo de manera privada una responsabilidad que compete a toda la sociedad (puesto que esos mayores también contribuyeron a que pudiésemos tener un Estado de bienestar). Estas decisiones se toman a nivel político asumiendo que "las familias", un eufemismo que de hecho suele implicar a las mujeres en mayor medida que a los varones, se harán cargo de aquello de lo que no puede ocuparse el Estado. Sin embargo, el Estado no regula el mercado laboral de tal modo que las personas empleadas tengan realmente tiempo para cuidar, de sí mismas o de otras personas. Creemos que, aunque es cierto que el cuidado no tiene por qué depender solo de las situaciones económicas, no podemos no tener en consideración, en nuestros análisis, las desigualdades socio-económicas que fomentan sesgos a la hora de ver respetado el derecho primordial a llevar una "vida buena".

Por ello, como científicas sociales, consideramos necesario fomentar el trabajo etnográfico para lograr un mayor conocimiento de cómo se articulan las relaciones de cuidado en función de los contextos concretos y de las relaciones de poder existentes en cada espacio social. Además, creemos que los estudios empíricos, a través de sus resultados, pueden ofrecer alternativas para reconfigurar también las políticas públicas, de modo que estas se centren en las necesidades de todas y todos, y respaldar las alternativas teóricas al actual discurso hegemónico edificado sobre los valores «universales» de la razón y del interés individualista/egoísta.

\section{Notas}

Este artículo es fruto de las investigaciones que hemos podido desarrollar con ayuda institucional. En el caso de Chiara Cerri, a través de la Ayuda Predoctoral de Formación de Personal Investigador BES2009-026934, y en el caso de Laura Alamillo Martínez, gracias al programa de Formación del Profesorado Universitario del Ministerio de Educación, referencia AP2008-00727.

1. Para ampliar el tema, se pueden consultar entre otras: Harris y Young 1979; Ortner y Whitehead 1981; Butler 1990, 2004; Moore 1991; Héritier 2000; Téllez Infantes 2001; Massó Guijarro 2004; Martín Casares 2006; Cerri 2010.

2. La expresión cuarta edad es utilizada para referirse a los mayores en una vejez avanzada, que habitualmente se considera comience a partir de los 80 años de edad, para diferenciarlo de la tercera edad, en la que se suelen agrupar los mayores de 65 a 80 años.

\section{Para ampliar esta cuestión acudir a Fox 2005}

4. Giddens (1995) desarrolla la idea de que la modernidad conlleva la reflexividad del individuo, que constantemente tiene que elaborar su propia biografía y sopesa qué implican para él las elecciones que toma.

5. Los Beck definen la individualización como "una condición social no alcanzable por libre decisión de los individuos. [...] Uno de los rasgos más decisivos de los procesos de individualización es, pues, que 
éstos no sólo permiten, sino que también exigen una activa contribución por parte de los individuos. A medida que se amplía la gama de opciones y que aumenta la necesidad de decidir entre ellas se hace mayor la necesidad de acciones realizadas individualmente, de ajuste, de coordinación, integración" (Beck y Beck-Gernsheim 2002: 42). La individualización indica entonces un proceso de posicionamiento y particularización del sujeto a través de su acción social dentro de la sociedad. Se distingue así del individualismo, concebido como formación de una individualidad totalmente autónoma, y de la individuación, que se refiere al proceso según el cual el individuo se individua a sí mismo en la intersección entre tiempo y espacio personal/colectivo.

6. Para más información respecto a la noción de familias electivas consultar Weston 2003.

7. Precarias a la deriva hablan de "externalización del hogar" (Precarias a la deriva 2005), mientras que Pérez Orozco y López Gil diferencian entre "externalización”, los trabajos de cuidados realizados fuera del hogar a través, sobre todo, de empresas privadas e instituciones públicas, y "mercantilización”, el hecho de "comprar" cuidado, fuera o dentro del hogar (Pérez Orozco y López Gil 2011).

8. Tronto diferencia cuatro fases y dimensiones del cuidado: Caring about (preocuparse de); Taking care of (o Caring for) (ocuparse de); Care giving (realizar cuidado); Care receiving (recibir cuidado) (Tronto 2005, 2009, 2010).

9. La ética del cuidado de Gilligan ha sido criticada por la asociación entre mujeres y cuidado que hacían de su teoría una "ética femenina del cuidado". Por ejemplo Tronto argumenta que si existe una diferencia entre moralidad femenina y masculina, esto no se debe a unas causas psicológicas, sino a unas causas culturales y sociales, como el estatus subordinado de las mujeres en la mayoría de las sociedades. Para responder a estas críticas, Gilligan posteriormente se preocupó de matizar que ella no entiende el cuidado como "natural" y propio de las mujeres, sino como una preocupación humana (Gilligan 2011).

10. Por "moral" nos referimos a las normas que guían los actos prácticos, la vivencia cotidiana de adhesión a principios y normas, con las que los actores juzgan los actos como correctos/incorrectos o justo e injusto. Por "ética" nos referimos a la reflexión teórica que se hace acerca de dichas normas morales, como una reflexión crítico-racional de la moral.

11. Seyla Benhabib cree que en vez de contraponer la ética del cuidado a la ética de la justicia sería más adecuado formular una teoría moral que mantenga el equilibrio entre particularismo y universalismo (Benhabib 1992).

12. Martín Palomo propone la "domesticación del trabajo" para superar la dualidad masculino/femenino y considerar las calidades y las condiciones de los trabajo desvinculando el cuidado y el trabajo doméstico del sujeto que lo realiza, las mujeres (Martín Palomo 2008b, 2009).

13. http://www.boe.es/boe/dias/2012/07/14/pdfs/BOE-A-2012-9364.pdf

14. http://www.boe.es/boe/dias/2012/04/21/pdfs/BOE-A-2012-5337.pdf

15. http://www.boe.es/boe/dias/2012/04/24/pdfs/BOE-A-2012-5403.pdf

\section{Bibliografía}

Aguirre, Rosario

2005 "Los cuidados familiares como problema público y objeto de políticas", comunicación presentada en Reunión de expertos CEPAL "Políticas hacia las familias, protección e inclusión sociales", Santiago, 28 y 29 de junio. 
Alamillo, Laura (y Cerri, Chiara)

2012 "Instituciones que son familias", comunicación presentada en XV Jornada de Antropología Ankulegi "Cuestiones contemporáneas sobre parentesco: nuevas perspectivas y problemáticas en el estudio antropológico de los lazos familiares", San Sebastián, 16 de marzo de 2012

Anderson, Jeanine

2010 Construyendo redes: las mujeres latinoamericanas en las cadenas globales de cuidado. Informe final. Mimeo.

Badgett, M.V. Lee (y Nancy Folbre)

1999 "¿Quién cuida de los demás? Norma sociosexuales y consecuencias económicas”, Revista Internacional del Trabajo, 118 (3): 347-365.

Balbo, Laura

1994 "La doble presencia", en Cristina Borderías; Cristina Carrasco y Carme Alemany (coord.), Las mujeres y el trabajo: rupturas conceptuales. Barcelona, Icaria: 503-514.

Bazo, María Teresa

2008 "Personas mayores y solidaridad familiar", Política y Sociedad, 45, 2: 73-85.

Beck, Ulrich (y Beck-Gernsheim, Elisabeth)

2002 Individualization: institutionalized individualism and its social and political consequences. London, SAGE.

Benhabib, Seyla

1992 "Una revisión del debate sobre las mujeres y la teoría moral”, Isegoría, 6: 37-63.

Berk, Sarah

1985 The gender factory: the apportionment of work in American households. Nueva York, Plenum.

Butler, Judith

1990 Gender trouble: feminism and the subversion of identity. New York, London, Routledge.

2004 Undoing Gender. New York, London, Routledge.

\section{Carrasco, Cristina}

1998 "Género y valoración social: la discusión sobre la cuantificación del trabajo de las mujeres", Mientras Tanto, 71. Barcelona, Icaria Editorial.

Carrasco, Cristina et al.

2003 Malabaristas de la vida. Mujeres, tiempos y trabajos. Barcelona, Icaria.

Cerri, Chiara

2010 " La subjetividad de género. El sujeto sexuado entre individualidad y colectividad", Gaceta de Antropología, 26/2, 42.

Comas d'Argemir, Dolors

1993 "Sobre el apoyo y el cuidado. División del trabajo, género y parentesco", en XavierRoigé i Ventura (coord.), Perspectivas en el estudio del parentesco y la familia. Tenerife, VI Congreso de Antropología: 65-82.

2000 "Mujeres, familia y Estado del bienestar", en Del Valle, Teresa (Coord.), Perspectivas feministas desde la antropología social. Madrid, Ariel: 187-204.

2009 "Trabajo, economía sumergida y género. La atención a la dependencia", en Anastasia Téllez A. y Javier Martínez Guirao (eds.), Economía informal y perspectivas de género en contextos de trabajo, Barcelona, Icaria: 169-195.

Daly, Mary (y Jane Lewis) 
2000. "The concept of social care and the analysis of contemporary Welfare State", British Journal of Sociology, 1: 281-298.

De Vault, Marjorie L.

1991 Feeding the family: the social organization of caring as gendered work. Chicago, University of Chicago press.

Del Valle, Teresa

2000 Perspectivas feministas desde la antropología social, Madrid, Ariel.

2004 "Contenidos y significados de nuevas formas de cuidado", Congreso Internacional Sare 2003:

Cuidar cuesta: costes y beneficios del cuidado: 39-61.

Durán, María Ángeles

2000 "Concentración y reparto del trabajo no remunerado en los hogares", Cuaderno de Relaciones Laborales, 17: 91-122.

2002 Los costes invisibles de la enfermedad. Bilbao, Fundación BBVA.

Edhom, Felicity (y Olivia Harris y Kate Young)

1977 "Conceptualizing Women", Critique of Anthropology, 3 (9-10).

Evers, Adalbert (y Pilj, Maarja. y Ungerson, Clare)

1994 Payments for care: a comparative overview. Adershot, Avebury Ed.

Ferree, Myra

1990 "Beyond separates spheres: feminism and family research", Journal of Marriage and the family, 52: 866-884.

Fine, Michael (y Carolina Glendinning)

2005 "Dependence, independence or inter-dependence? Revisiting the concepts of 'care' and 'dependency"', Ageing \& Society, 25: 601-621.

Fischer, Berenice (y Joan Tronto)

1990 "Toward a Feminist Theory of Caring", en Emily Abel y Margaret Nelson(eds.), Circles of Care. Albany, State University of New York Press: 36-54.

Fox, Robin

2005 Sistemas de parentesco y matrimonio. Madrid, Alianza Universidad.

Garrau, Marie (y Le Goff, Alice)

2010 Care, justice et dépendance. Introduction aux théories du Care. Paris, Presses Universitaires de France.

Giddens, Anthony

1995 Modernidad e identidad del yo. El yo y la sociedad en la época contemporánea. Barcelona, Ediciones Península.

Gilligan, Carol

1982 In a different voice. Psychological Theory and Women'sDevelopment. Harvard University Press, Cambridge.

2011 "Une voix diferente. Un regard prospectif à partir du passé", en Patricia Paperman y Sandra Laugier (eds.), Le Souci des autres. Éthique et politique du care. Paris, École des Hautes Études en Sciences Sociales: 37-50.

Grasmuk, Sherri (y Patricia Pessar)

1991 Between two islands: dominican international migration. Berkeley, University of California Press. 
Gregorio Gil, Carmen

2005 "La provisión de bienestar y las "obligaciones" de género y parentesco: una propuesta de análisis en el contexto local/global", comunicación presentada en VI taller internacional ¿mujeres en el siglo XXI?, La Habana, 21-25 de noviembre.

Guérin, Serge

2010 "Politique du don, politique du care. Stratégie de la société accompagnante", La Revue, n 4

Guzmán Castillo, Francisco (y Toboso Martín, Mario y Romañach Cabrero, Javier)

2010 "Fundamentos éticos para la promoción de la autonomía: hacia una ética de la interdependencia", Alternativas, 17: 45-61.

Haber, Stéphane

2011 "Éthique du care et problématique féministe dans la discussion américaine actuelle. De C. Gilligan à J. Tronto", en Patricia Paperman y Sandra Laugier (eds.), Le Souci des autres. Éthique et politique du care. Paris, École des Hautes Études en Sciences Sociales: 187-208.

Harris, Olivia (y Kate Young )

1979 Antropología y feminismo. Barcelona, Anagrama.

Héritier, Françoise

2000 Masculino/femenino. El pensamiento de la diferencia. Barcelona, Ariel.

Himmelweit, Susan

1995 "The Discovery of "Unpaid Work": The Social Consequences of the Expansion of "Work", Feminist Economics, 1(2).

Hochschild, Arlie Russell

2003 The commercialization of intimate life: notes from home and work. University of California Press, Berkeley.

Hondagneu-Sotelo, Pierrette

1992 "Overcoming Patriarchal Constraints: the reconstruction of gender relations among Mexican immigrant women and men", Gender and Society, 6: 393-415.

1994 Gendered transitions. Mexican experiences of migration. California, University of California Press.

Kittay, Eva Feder

2002. The Subject of Care. Feminist Perspectives on Dependency. Lanham, Rowman and Littlefield Publishers.

Kolberg Lawrence

1963 "The development of children's orientations toward a moral order: I. Sequence in the development of moral thought", Vita Humana, 6: 11-33.

1981 Essays on moral development. Nueva York, Harper and Row.

Leira, Arnlaug (y Chiara Saraceno)

2002. "Care: actors, relationship and contexts", en Barbara Hobso, Jane Lewis y Birte Siim (eds.), Contested concepts in gender and social politics. Chelterham, Edward Elgad.

Martin, Claude

2008 "Qu'est-ce que le social care? Une revue de questions", Revue Française de Socio-Économie, 2008/2, n²: 27-42.

Martín Casares, Aurelia

2006 Antropología del género. Culturas, mitos y estereotipos sexuales. Madrid, Ediciones Cátedra. 
2008a "Los cuidados y las mujeres en las familias", Política y Sociedad, 45, 2: 29-47.

2008b "Domesticar el trabajo: una reflexión a partir de los cuidados", Cuadernos de relaciones laborales, 26, 2: 13-44.

2009 "El care, un debate abierto: de las políticas de tiempo al social care", Cuestiones de género: de la igualdad y la diferencia, 4: 325-355.

Massó Guijarro, Ester

2004 ” Género y ciencia. Una relación fructífera “, Gaceta de Antropología, 20, 6.

Molinier, Pascale

2009. "Vulnérabilité et dépendance: de la maltraitance en régime de gestion hospitalière", en Marlène Jouan y Sandra Laugier (eds.), Comment penser l'autonomie? Entre compétence et dépendances. Paris, Puf: 433-458.

Moore, Henrietta

1991 Antropología y feminismo. Valencia, Universitat de València.

Narotzky, Susana

2004 Antropología Económica. Nuevas Tendencias. Barcelona, Editorial Melusina.

Ortner, Sherry (y Whitehead Harriet)

1981 Sexual meanings. The cultural construction of gender and sexuality. Cambridge, Cambridge University Press.

Paperman, Patricia (y Sandra Laugier)

2011 Le Souci des autres. Éthique et politique du care, Paris: École des Hautes Études en Sciences Sociales.

Paperman, Patricia

2011 "Les gens vulnérables n'ont rien d'exceptionnel", en Patricia Paperman y Sandra Laugier (eds.), Le Souci des autres. Éthique et politique du care, Paris: École des Hautes Études en Sciences Sociales: 321-337.

Pattaroni, Luca

2011 "Le care est-il institutionnalisable? Quand la «politique du care» émousse son éthique", en Patricia Paperman y Sandra Laugier (eds.), Le Souci des autres. Éthique et politique du care. Paris, École des Hautes Études en Sciences Sociales: 209-233.

Pérez Orozco, Amaia

2006 "Amenaza tormenta: la crisis de los cuidados y la reorganización del sistema económico", Revista de Economía Crítica, 5: 7-37.

Pérez Orozco, Amaia (y Silvia López Gil)

2011 Desigualdades a flor de piel: cadenas globales de cuidados. Concreciones en el empleo de hogar y políticas públicas. Madrid: ONU Mujeres.

Pichardo, Ignacio

2009 "(Homo)sexualidad y familia: cambios y continuidades al inicio del tercer milenio", Política y Sociedad, 46, 1 y 2: 143-160.

Precarias a la deriva

2004 A la deriva por los circuitos de la precariedad femenina. Madrid, Traficantes de sueños.

2005 Una huelga de mucho cuidado. Cuatro hipótesis.

http://www.sindominio.net/contrapoder/article.php3?id_article= [Consulta 10 de noviembre de 2011]. 
Rapp, Rayna

1978 "Family and Class in Contemporary America: notes Toward an Understanding of Ideology", Science and Society, 42 (3).

1987 "Toward a Nuclear Freeze? The Gender Politics of Euro-American Kinship Analysis", en Jane Collier y Sylvia Yanagisako (eds.), Gender and Kinship: Essays Toward a Unified Analysis. Standford, California, Standford University Press

Rivas, Ana María (y Herminia Gonzálvez y Cristina Gómez)

2009 "Los enfoques teóricos", en Ana María Rivas y Herminia Gonzálvez (eds.) Familias Transnacionales Colombianas. Transformaciones y permanencias en las relaciones familiares y de género. Catarata, Madrid.

Saillant, Francine (y Éric Gagnon)

2001 "Responsabilité pour autrui et dépendance dans la modernité avancée. Le cas de l'aide aux proches", Lien social et Politiques, 46: 55-69.

Saraceno, Chiara

2005 “¿Qué derecho y obligaciones, qué tipos de recursos? Visiones de la ciudadanía a través del prisma del género", en Congreso Internacional Sare 2004: ¿Hacia que modelo de ciudadanía?: 255-278. Vitoria, EMAKUNDE (ed.), Instituto Vasco de la Mujer.

Schafer, Dieter

1995 "La producción doméstica en Alemania: conceptos y planes para un sistema de contabilidad satélite", Política y Sociedad, 19: 33-43.

Téllez Infantes, Anastasia

2001 " Trabajo y representaciones ideológicas de género. Propuesta para un posicionamiento analítico desde la antropología cultural", Gaceta de Antropología, 17, 17.

Tronto, Joan

2005 "Cuando la ciudadanía se cuida: una paradoja neoliberal del bienestar y la desigualdad", en Congreso Internacional Sare 2004 ¿Hacia que modelo de ciudadanía?: 231-253. Vitoria, EMAKUNDE (ed.), Instituto Vasco de la Mujer.

2009 Un monde vulnerable. Pour une politique du care. Paris, Éditions la Découverte.

2010 "Cura e politica democratica. Alcune premesse fondamentali", La societá degli individui, 38 (2): 34 42.

2011 "Au-delà d'une différence de genre. Vers une théorie du care", en Patricia Paperman y Sandra Laugier (eds.), Le Souci des autres. Éthique et politique du care. Paris, École des Hautes Études en Sciences Sociales: 51- 77.

Vega Solís, Cristina

2009 Culturas del cuidado en transición. Barcelona, Editorial UOC.

Willis, Paul

2007 "Producción cultural no es lo mismo que reproducción cultural, que a su vez no es lo mismo que reproducción social, que tampoco es lo mismo que reproducción", en Franciso J. García Castaño, Honorio M.Velasco y Ángel Díaz de Rada (coords.), Lecturas de Antropología para Educadores. El ámbito de la antropología de la educación y de la etnografía escolar. Madrid, Trotta.

Weston, Kath

2003 Las familias que elegimos: lesbianas, gays y parentesco. Bellaterra, Barcelona. 
